\title{
LA CULTURA COMO MOTOR DE DESARROLLO TURÍSTICO: INVENTARIO DEL PATRIMONIO Y DISEÑO DE LA RUTA TURÍSTICA "MORÓN OCULTO"
}

Germán Ramos Campos ${ }^{1}$

germanramos19@msn.com

(Fecha envío: 23/04/17 - Fecha aceptación: 18/05/17)

\section{Resumen}

En este artículo planteamos que el desarrollo turístico no debería concebirse al margen de la cultura, ya que ésta es el principal mecanismo para el desarrollo social y económico de los pueblos.

De acuerdo con lo anterior, en este estudio se desarrolla un inventario patrimonial de Morón de la Frontera, teniendo presente los valores históricos, artísticos y etnológicos, y atendiendo a sus dimensiones materiales e inmateriales. Este inventario es el instrumento para el diseño de una ruta turística que trata de articular los distintos elementos patrimoniales de la localidad. Las arraigadas leyendas locales constituirán el hilo conductor de este itinerario que transitará por los hitos patrimoniales más significativos de Morón de la Frontera.

\section{Palabras Clave}

cultura, turismo, Morón, inventario, ruta

\section{Abstract}

In this paper, we propose that tourism development should not be seen outside the culture, as this is the main mechanism for social and economic development of peoples.

According to the above, in this study a heritage inventory of Morón de la Frontera is developed, taking into account the historical, artistic and ethnological values, and serving their tangible and intangible dimensions. This inventory is the instrument for designing a tourist route that tries to articulate the various assets of the locality. Entrenched local legends constitute the theme of this itinerary that will transit the most significant heritage landmarks of Morón de la Frontera.

\section{Keywords}

culture, tourism, Morón, inventory, route

\section{INTRODUCCIÓN}

La cultura está ligada estrechamente a la actividad turística, en cualquier tipo de turismo siempre hay contacto con la cultura receptora. Este contacto se incrementa a medida que el patrimonio y las tradiciones y costumbres de una zona determinada se tienen en cuenta como recursos para el despliegue turístico de ésta.

La cultura confiere al turismo un valor añadido, aportando numerosos activos, no solo bienes muebles e inmuebles, sino también usos y costumbres, gastronomía, ritos y leyendas que contribuyen al desarrollo socioeconómico del entorno, de las empresas o de los individuos. En definitiva, la cultura en el turismo no es solo un medio para el desarrollo turístico, sino que se convierte en un fin en sí mismo indispensable para el despegue turístico de una zona.

El turismo es un fenómeno social que manifiesta un crecimiento constante, constituyendo una notable fuente de riqueza económica y sociocultural. El turista pretende gozar de una experiencia enriquecedora y personalizada; por ello, concibe el viaje como una inversión productiva dados los beneficios intelectuales, morales y sociales que a éste se vinculan. Además, el interés por la cultura, la nostalgia por el pasado y la autenticidad presente en la sociedad actual conducen a redescubrir las diferencias culturales de los pueblos.

Con respecto a la metodología seguida hemos contado con instrumentos cualitativos y cuantitativos para elaborar un diagnóstico preciso. Se ha desarrollado un exhaustivo trabajo de campo con el propósito de obtener la información necesaria que posibilitara la elaboración de un diagnóstico turístico bien estructurado y de un inventario holístico en el que se tenga en cuenta todos los parámetros del recurso. Para ello hemos contado con

\footnotetext{
${ }^{1}$ Máster en Dirección y planificación del Turismo
} 
los responsables de la Administración local relacionados directamente con la actividad turística de Morón, así como con los empresarios del sector y otros agentes implicados.

\section{LA CULTURA COMO MOTOR DE DESARROLLO TURÍSTICO}

\subsection{Cultura y patrimonio}

Según la Declaración sobre los Derechos Culturales (declaración de Friburgo,2007), la cultura es "el conjunto que comprende los modos de vida, las instituciones, las tradiciones, los saberes y artes, la lengua, las creencias, los valores y las expresiones mediante los que un pueblo, expresa los significados que otorga su existencia". Por otra parte, el uso convencional que se hace de la cultura se refiere a la formación académica del individuo, por lo que podría definirse como aquel cúmulo de conocimientos superiores que se tiene acerca de diferentes materias y permite exponer o desarrollar juicios críticos.

Atendiendo a este concepto existen individuos que tienen cultura y otros que no, mientras que según el concepto de cultura empleado por la antropología social, todos los individuos poseemos cultura, no por el hecho de ser individuos, si no como miembros de una sociedad; por lo que cabría destacar entre ellos una diferencia fundamental: la cultura tal y como es entendida por la antropología social comprende además de conocimientos, modos de vida, costumbres, creencias, valores... y la cultura como concepto de uso convencional, solamente se asocia al conocimiento, al nivel de instrucción.

Con respecto a la diferencia entre cultura y patrimonio cultural, por un lado, mientras que la Cultura en sí misma, comprende los modos de vida de una sociedad, sus costumbres, conocimientos, tradiciones, etc., el patrimonio cultural sería un elemento de la cultura. Podría definirse como un conjunto de bienes que la sociedad tiene en alta estima, ya que representa la historia de esta, sus tradiciones, sus modos de vida... Podría decirse que el patrimonio cultural es una construcción cultural, algo así como un elemento existente dentro de la cultura.

Según Isidoro Moreno en su artículo "Cultura andaluza, patrimonio cultural y políticas del patrimonio" (2002, pág. 90), pueden diferenciarse tres ámbitos diferentes del patrimonio cultural: el patrimonio declarado legalmente tal por la administración; el patrimonio definido por los "especialistas en patrimonio" y por los profesionales de las diversas disciplinas que inciden en él; el patrimonio realmente percibido y vivido por los distintos grupos y colectivos sociales.

Como dice el Antropólogo Javier Hernández-Ramírez (2011, pág. 230), el patrimonio se puede entender por un lado desde el punto de vista de una tesis restrictiva y por otro teniendo en cuenta una tesis aperturista. La tesis restrictiva limita la calificación de patrimonio a objetos materiales concretos que son seleccionados por expertos académicos según 4 criterios definidos: materialidad, antigüedad, excepcionalidad y estética. En la tesis aperturista el patrimonio comprende también las manifestaciones culturales intangibles, como son las tradiciones orales, la música, las festividades o las lenguas. Cada bien cultural constituye una riqueza colectiva, cuyo valor lo proporciona su reconocimiento, valoración y estima social.

Siempre hemos tenido la tendencia natural de asociar la palabra patrimonio con algo material, pero como hemos podido comprobar, también existe un tipo de patrimonio formado en gran parte por elementos intangibles, inmateriales, en definitiva, lo que hoy conocemos por patrimonio etnológico.

Por un lado, el término patrimonio hace referencia a aquello que heredamos, pero por otro lado, la palabra patrimonio engloba mucho más.

Con respecto al patrimonio etnológico, es uno de los patrimonios más olvidados, además de ser el menos valorado por las instituciones, relegándolo a un segundo plano. Esto acelera el riesgo que tiene el patrimonio etnológico de desaparecer

Hace falta un conocimiento de las técnicas de trabajo de los oficios tradicionales, ya que si no se terminarán perdiendo, como podría haber pasado en Morón de la Frontera con el oficio del calero, que si no hubiera sido por un grupo de personas, hoy día no se conocería este oficio, y la labor que desarrollaban, tan tradicional en nuestra comunidad autónoma. Pero este grupo de personas ha sabido canalizar la importancia patrimonial de la cal y ha conseguido la máxima declaración y máxima protección para que perviva siempre este oficio tradicional y toda la connotación social que éste conlleva en la zona.

El patrimonio etnológico es parte fundamental de nuestro legado y si no valoramos lo que nos ha sido transmitido de generación en generación desaparecerá una parte de nuestra historia y se acabaran perdiendo nuestras tradiciones.

El conocimiento y compromiso que manifiesten en la preservación del patrimonio; a mi entender debieran ser justamente las comunidades locales los principales gestores del patrimonio y por supuesto es responsabilidad de 
las Universidad modular diferentes acciones de investigación y formación para contribuir a la gestión del patrimonio.

El patrimonio intangible está presente en todos los elementos del patrimonio cultural. Hoy día debemos plantearnos integrar mejor la protección del patrimonio y la valorización del patrimonio dentro de la perspectiva local de desarrollo teniendo en cuenta todos sus parámetros. Probablemente algo que nosotros entendemos como bien patrimonial en nuestro entorno, ya sea por arraigo, tradición o por un valor dado, puede que en otro sitio no tenga ningún interés y no sea valorado, ni le preste mayor atención, pero no por ello dejaría de ser patrimonio el primero de los casos planteado.

El concepto de Patrimonio Cultural ha ido cambiado a lo largo de los años. Hoy día, aquello que se considera Patrimonio, se intenta proteger para que, aparte de poder sacarle rendimiento, (en mayor parte económico), también puedan disfrutarlo las generaciones futuras; mientras que en el siglo XIX, por ejemplo tal como señala Antonio Limón en su artículo "Patrimonio, ¿De quién? (1999, pág. 9), se derribaron las murallas de algunas ciudades como Sevilla, y no estaba mal considerado por la sociedad.

Para la sociedad es muy fácil reconocer un objeto y asociarlo a que es escaso por su rareza, su belleza, su antigüedad... Sin embargo, asociar el Patrimonio Cultural a Bienes Inmateriales, parece que actualmente, es algo más complicado de entender para la sociedad, ya que en estos no se puede concebir su escasez, aunque en los últimos años se ha dado un paso importante y el patrimonio inmaterial es reconocido por la sociedad y protegido por las instituciones.

Según Antonio Limón existen entendidos de la materia, cuyo único afán es contraponer los Bienes materiales contra los Bienes Inmateriales, y esto no tiene ningún sentido, ya que según este autor "es inseparable el martillo del golpe". Esto quiere decir, que el martillo, no podría definirse sin el golpe que produce; metáfora que representa que los Bienes Intangibles no pueden precisarse sin los Bienes materiales y viceversa.

En este artículo se lleva a cabo una identificación del patrimonio de la localidad de Morón de la Frontera y una propuesta de itinerario o ruta turística. Precisamente el objetivo que subyace a estas actividades es contribuir a una mayor conciencia de la ciudadanía nativa y forasteras sobre el valor de la cultura y el patrimonio.

\subsection{El turismo cultural}

Para la Organización Mundial del Turismo (OMT), el turismo cultural es definido como el "movimientos de personas debido esencialmente a motivos culturales como viajes de estudio, viajes a festivales u otros eventos culturales, visitas a sitios y monumentos, viajes para estudiar la naturaleza, el arte o el folklore, y peregrinaciones" (Pedersen, 2005, OMT).

De otro lado, Toselli (2007, pag 178) argumenta que la preservación del patrimonio también debe darse desde la participación del turista, partiendo del hecho de fomentar en ellos la sensibilidad por el cuidado del patrimonio y respeto de las culturas de las comunidades visitadas. Aquí no solamente basta con que el turista llegue al destino y se aloje en una finca, consuma los productos típicos de la zona local y tome fotos que posteriormente serán publicadas en las redes sociales. No se trata del consumo turístico por sí solo, es necesario que el turista conozca la historia de los platos típicos, así como la importancia que representan las actividades que se desarrollan en la finca. Es decir, se requiere de la identificación del turista con las tradiciones locales.

Para el buen desarrollo turístico de una zona es necesario promover el interés de los habitantes por la cultura a través de sus costumbres, artesanías, folklore, fiestas, gastronomía y tradiciones; además se debe fomentar en los turistas la sensibilidad por el cuidado del patrimonio y respeto de las culturas en las zonas visitadas.

\subsection{Gestión turística del patrimonio cultural}

El turismo cultural puede convertirse en un instrumento positivo tanto para la revalorización del patrimonio cultural como para el desarrollo económico de una zona.

Por lo que respecta a la gestión del patrimonio cultural, tradicionalmente, se ha obviado el papel fundamental que el turista juega en todo el proceso, que cada vez está más informado, es más exigente y valora su experiencia turística realizando un análisis coste-beneficio. Por ello, al tomar en consideración todas estas circunstancias los gestores se encuentra con la tarea de educar a la demanda a través de un acercamiento entre patrimonio y turista, explicándoles las características particulares del patrimonio tangible e intangible desde una perspectiva amena, sencilla, atractiva y cómoda. Los bienes del patrimonio histórico han pasado a considerarse como productos turísticos pues se entiende que el objetivo perseguido con su lanzamiento al mercado es el suministro de una experiencia enriquecedora que satisfaga las necesidades de los turistas (Mckercher and Ho, 2004, pág. 256).

La gestión turística del patrimonio cultural según Toselli puede revitalizar el interés de los habitantes por su cultura, así como en la protección del patrimonio arquitectónico y artístico y contribuir a atenuar o romper la 
estacionalidad en destinos cuya oferta principal se base en productos de sol y playa. Generar recursos para el mantenimiento, protección y mejora de los sitios de patrimonio y recuperar viejos recursos para nuevas formas de turismos, puesto que a través de la puesta en valor de recursos del patrimonio tangible e intangible se recuperará la herencia de los antepasados y se integrarán en los proyectos de desarrollo local a través del turismo.

No obstante, también puede tener efectos no deseados los cuales deben ser minimizados en la medida de lo posible por los agentes implicados, entre estos riesgos Toselli destaca el deterioro de la cultura del destino a través de la producción y venta de artesanías no auténticas o el desarrollo de fiestas que constituyen una puesta en escena alejada de la realidad, generar un sentimiento de rechazo por parte de las comunidades visitadas cuando no se respetan los sitios o las costumbres del lugar e impulsar la mercantilización extrema de las tradiciones locales, despojándolas de su verdadero significado, convirtiendo la cultura local en un mero objeto de consumo.

Todo lo anterior hace de la cultura un bien de consumo de primer orden, ya sea como motivo principal o secundario del viaje. Por lo tanto, se debe hacer una oferta de calidad que no solo potencie los beneficios que produce, sino que minimice los riesgos que conlleva.

En este sentido, la propuesta de ruta que en este artículo se diseña tiene como fundamento la perspectiva emic del patrimonio, es decir, la visión nativa, conectando las leyendas y las tradiciones con el patrimonio histórico, artístico y etnológico presente en las calles moroneras.

\section{UNIDAD DE OBSERVACIÓN}

\subsection{Morón de la Frontera}

La ciudad de Morón de la frontera se encuentra enclavada en la Serranía Suroeste Sevillana entre la Campiña y las Sierras Subbéticas. Zona rica por sus paisajes, donde amplias Ilanuras onduladas se mezclan con las primeras estribaciones del borde meridional del valle del Guadalquivir. Los olivares bañan sus campos y los girasoles y el trigo dibujan un paisaje lleno de contrastes. Cuenta con un clima mediterráneo con temperaturas suaves en invierno y veranos calurosos. Un territorio principalmente agrícola donde grandes llanuras y encrespadas sierras sitúan la localidad en un lugar estratégico.

Morón Cuenta con una población de 28.467 habitantes (2010) en una extensión superficial de 431,94km². Morón se encuentra a una altitud de 297 metros sobre el nivel del mar y a 62 kilómetros de la capital.

Con respecto a la economía, antes de finalizar el siglo XIX se produjeron tres hechos relevantes: la inauguración de la línea ferroviaria Morón-Sevilla (1.864), la salida a la luz del primer periódico local impreso La Razón (1.885) y la concesión a esta villa del título de Ciudad por la Reina María Cristina (1.894).

Desde finales del siglo XIX hasta las primeras décadas del XX, proliferaron algunas industrias de aceite, aceitunas, cemento y cal, contribuyendo al desarrollo económico e industrial de Morón. En los años 40 y 50 , la construcción de la Base Aérea Española (1.944) y americana (a partir de 1.956) atrajo mucha mano de obra especializada, provocando un considerable aumento de la población, que llegó a rebasar los 37.000 habitantes.

Entre sus edificaciones de interés histórico artístico destacan las iglesias de San Miguel (siglos XVI-XVIII), San Francisco (siglo XVI), Nuestra Señora de la Victoria (siglo XVI), San Ignacio (siglo XVIII), la Merced (siglo XVIII), Santa María de la Asunción, Santa Clara y San Juan de Dios (siglo XVII), la ermita de Nuestro Padre Jesús de la Cañada (siglo XVIII), la Cilla del Cabildo, así como los restos de murallas y la Torre del Homenaje. También tenemos que tener en cuenta el patrimonio etnológico, es decir, sus fiestas, la artesanía, el flamenco, etc. En el territorio objeto de estudio existe una gran variedad de patrimonio etnológico: Romería de Gracia, el flamenco en Esparteros, la rica tradición oral, la arquitectura tradicional y como no, la artesanía de la cal.

\section{PATRIMONIO CULTURAL DE MORÓN DE LA FRONTERA}

\section{1. ¿Qué entendemos por inventario?}

El primer paso para llevar a cabo una efectiva protección del patrimonio cultural es el conocimiento. La investigación determina la mayor o menor dimensión cultural de los bienes culturales. La investigación del patrimonio comienza con la identificación de los bienes culturales existentes en una determinada zona. Esta documentación es la materia prima de los inventarios.

Un inventario consiste en contar y ordenar con precisión aquellos bienes pertenecientes a una persona o a una comunidad. En el caso del patrimonio se trata de identificar los elementos más sobresalientes y valorados pertenecientes a una comunidad simbólica de individuos. 
Debe existir sin duda alguna, un tipo de inventario que sea capaz de recoger la riqueza patrimonial existente, y servir como paso previo a cualquier tipo de legislación de protección.

Según Agudo, J, "no solo hay que inventariar como proceso técnico previo a cualquier medida de protección, también hay que inventariar para conocer la realidad del patrimonio sobre el que queremos intervenir", (1999, pág.53). Esta afirmación nos demuestra que los inventarios no deben realizarse solamente para conseguir enumerar y más tarde proteger todos los Bienes que una persona o comunidad posea, sino también, para poder transmitir el valor y el significado que los Bienes poseen, y por lo tanto, también poseen dichas personas 0 comunidades.

El inventario es útil para documentar, para conocer, para valorizar e investigar los Bienes Materiales, y gracias al conocimiento que se obtenga de estos, se podrán llegar a conocer los Bienes Etnológicos que se le relacionen. Ambos tipos de Bienes han de ir siempre de la mano.

Es preciso destacar, que el procesamiento de la información para el inventario es un trabajo permanente, de tal manera que éste se encuentre siempre actualizado. Un inventario tiene que ser claro, conciso y dinámico; permitiendo la actualización de los recursos turísticos.

Para la realización del inventario de recursos turísticos de Morón de la Frontera nos centraremos en primer lugar en ordenar y clasificar los monumentos, tradiciones y acontecimientos de la localidad para su posterior evaluación y jerarquización, que comprenderá el proceso de ponderación de los recursos, con el fin de determinar el valor y la importancia de cada uno de ellos.

Para entender bien la clasificación patrimonial propuesta es necesario conocer la protección actual del patrimonio a nivel mundial y a nivel autonómico, ya que la Junta de Andalucía tiene transferidas las competencias para la protección del patrimonio. Por ello en el siguiente apartado vamos a presentarlos diferentes rangos de protección ateniéndonos principalmente a la UNESCO y a la ley que se encarga de tutelar el patrimonio cultural en nuestra comunidad.

\section{2. ¿Cómo proteger el patrimonio?}

Los bienes inventariados pueden formar parte de catálogos oficiales, en los que una determinada administración adquiere el compromiso público de proteger aquellos bienes inscritos. A nivel internacional destacan la lista de Patrimonio Mundial y la lista representativa del patrimonio inmaterial de la Humanidad. Con respecto a este segundo catalogo Morón cuenta con dos bienes declarados Patrimonio inmaterial Cultural de la Humanidad, por un lado la Cal de Morón, con el título: "Revitalización del Saber Tradicional de la Elaboración de la Cal Artesanal en Morón de la Frontera, Sevilla, Andalucía” y por otro El flamenco, que aunque ocupa todo el territorio andaluz, está muy arraigado en la localidad.

La comunidad Andaluza tiene transferidas todas las competencias para la tutela del Patrimonio histórico, ésta las regula a través de la ley 14/2007, de 26 de noviembre, la cual concibe como instrumento fundamental para la tutela y conocimiento de los bienes el Catálogo General del Patrimonio Histórico Andaluz, y dentro del catálogo encontramos clasificados los bienes en tres categorías:

\begin{tabular}{|l|l|}
\hline Catálogo General & Bienes de Catalogación General \\
\cline { 2 - 2 } del Patrimonio Histórico Andaluz. & Inventario General de Bienes Muebles \\
\cline { 2 - 2 } CGPHA & Bienes de Interés Cultural (BIC) \\
\hline
\end{tabular}

Tabla 1: Catálogo General del Patrimonio Histórico Andalu. Fuente: Elaboración propia a partir de Ley 14/2007

\subsection{Inventario del patrimonio cultural de Morón}

Para la realización del inventario en primer lugar nos centraremos en aquellos bienes que tienen la máxima catalogación y están declarados Patrimonio de la Humanidad y en segundo lugar en aquellos que están incluidos en el Catálogo General del Patrimonio Histórico Andaluz, destacando aquellos que están declarados BIC. Para la realización de nuestro inventario hemos tenido en cuenta la misma tipología utilizada para la clasificación de bienes que se ciñe al artículo 25 del capítulo 1 de clasificación y ámbito de los Bienes de Interés Cultural de la Ley 14/07 de Patrimonio Histórico de Andalucía:

Dentro de los bienes patrimoniales de Morón, cabe destacar la inscripción de seis BIENES DE INTERES CULTURAL en el Catálogo General de Patrimonio Histórico de Andalucía, cinco en la categoría de MONUMENTO y uno en la categoría de INTERÉS ETNOLÓGICO. En la siguiente tabla se puede observar el año de inscripción y publicación de cada uno de ellos. 


\begin{tabular}{|c|c|c|c|c|}
\hline \multicolumn{5}{|c|}{ Iglesia de San Miguel } \\
\hline Estado & Régimen & Tipología jurídica & Publicado en & Fecha \\
\hline Inscrito & $\mathrm{BIC}$ & Monumento & BOE & $14 / 04 / 1970$ \\
\hline \multicolumn{5}{|c|}{ Casa de la Cultura } \\
\hline Estado & Régimen & Tipología Jurídica & Publicado en & Fecha \\
\hline Inscrito & $\mathrm{BIC}$ & Monumento & $\mathrm{BOE}$ & 04/12/1981 \\
\hline \multicolumn{5}{|c|}{ Castillo } \\
\hline Estado & Régimen & Tipología Jurídica & Publicado en & Fecha \\
\hline Inscrito & $\mathrm{BIC}$ & Monumento & $\mathrm{BOE}$ & 29/06/1985 \\
\hline \multicolumn{5}{|c|}{ Torre de la Atalaya } \\
\hline Estado & Régimen & Tipología Jurídica & Publicado en & Fecha \\
\hline Inscrito & $\mathrm{BIC}$ & Monumento & $\mathrm{BOE}$ & 29/06/1985 \\
\hline \multicolumn{5}{|c|}{ Iglesia de San Francisco } \\
\hline Estado & Régimen & Tipología Jurídica & Publicado en & Fecha \\
\hline Inscrito & $\mathrm{BIC}$ & Monumento & BOJA & $27 / 09 / 2001$ \\
\hline \multicolumn{5}{|c|}{ Caleras de la Sierra } \\
\hline Estado & Régimen & Tipología Jurídica & Publicado en & Fecha \\
\hline Inscrito & $\mathrm{BIC}$ & Lugar de Interés Etnológico & BOJA & 27/07/2009 \\
\hline
\end{tabular}

Tabla 2: Bienes BIC de Morón

Fuente: Elaboración propia a partir de Catálogo General del Patrimonio Histórico Andaluz

Para proteger el patrimonio debemos tener en cuenta la dimensión etnológica de los bienes (usos y significados). Por ejemplo, si visitas una iglesia, debemos tener en cuenta que estás viendo una iglesia del S.XXI aunque date del S.XVI. Se trata de comprobar los valores patrimoniales que atesoran cada bien y el conjunto de bienes de Morón. Por ejemplo, un historiador del arte sólo se fijaría en la dimensión artística de una iglesia, el historiador destacaría la antigüedad del templo, pero nosotros también nos hemos fijado en los usos sociales de la iglesia: de hermandades, centro de ceremonias, centro de la vida social en la plaza de al lado. También nos interesa mucho el significado simbólico del bien para los vecinos. Por ejemplo, la Giralda es una torre renacentista sobre alminar almohade, pero para los sevillanos es el símbolo de la ciudad. Todo bien tiene una visión material y otra inmaterial que aportan valores del bien.

A continuación, vamos a presentar el modelo de ficha diseñado para el inventario, plasmando en este estudio aquellos bienes más relevantes.

4.4. Modelo ficha de recopilación de datos para información del inventario de recursos turísticos de Moron de la Frontera

NOMBRE DEL BIEN PATRIMONIAL:

UBICACIÓN:

CATEGORÍA:

TIPOLOGÍA JURÍDICA:

CATALOGACIÓN:

DESCRIPCION:

USOS SOCIALES:

PARTICULARIDADES:

ESTADO ACTUAL:

OBSERVACIONES:

EPOCA PROPICIA DE VISITA AL RECURSO:

HORARIO DE VISITA:

PRECIO:

PROPIEDAD DEL RECURSO TURÍSTICO:

ADMINISTRADO POR:

MATERIAL AUDIO VISUAL ACTUAL DEL RECURSO TURISTICO:

RUTAS:

\section{PROPUESTAS DE DESARROLLO TURÍSTICO}

\subsection{La ruta como producto turístico}

Para realizar un proyecto turístico ambicioso es necesario aprender a gestionar los recursos en conjunto y no individualmente. Por ello en este proyecto apostamos por un modelo holístico de colaboración público-privada. 
En los últimos años el turista que visita bienes culturales parece estar mucho más motivado por aspectos intangibles de la experiencia turística urbana que por los elementos tangibles en sí. Una de las principales motivaciones del turista cultural es "experimentar". Apostamos desde estas líneas por un turismo participativo frente a un turismo meramente contemplativo.

Se considera como ruta turística un itinerario seguido a partir de un sitio de partida en una determinada dirección, para recorrer un espacio que tiene un sitio final o una llegada, lo que le permitirá percibir una experiencia de viaje. Briedenhann y Wickens la definen como la "creación de un cluster de actividades y atracciones que incentivan la cooperación entre diferentes áreas y que sirven de vehículo para estimular el desarrollo económico a través del turismo" (2003, pág. 57).

Las rutas deben crearse en función a un producto cultural característico de una zona, en el que el objetivo principal sea incentivar la realización de la ruta para así promover al desarrollo local donde transcurre la ruta.

En los últimos años las rutas turísticas han intentado innovar, lo que ha provocado una mayor exigencia por parte de la demanda en cuanto a la diversificación de la oferta. Por ello, es necesario dar un valor añadido a los productos para así lograr un mejor posicionamiento de los destinos turísticos.

Es importante destacar aquellos testimonios históricos, artísticos y etnológicos más relevantes para la consecución de un itinerario cultural que sea atractivo y auténtico para el turista. Los valores añadidos son primordiales, no podemos ceñirnos a lo que se puede ver o palpar, debemos tener una visión holística del lugar, para que los turistas revivan lo que aconteció en el pasado sin perder de vista el presente y disfrutando de la ruta de una forma completa.

\begin{tabular}{|c|l|}
\hline \multicolumn{1}{|c|}{ Rutas } & \multicolumn{1}{c|}{ Subtipos } \\
\hline \multirow{4}{*}{ Específicas Patrimoniales } & Rutas del Patrimonio Etnológico \\
\cline { 2 - 2 } & Rutas del Patrimonio Histórico y/o arqueológico \\
\cline { 2 - 2 } & Rutas del Patrimonio Natural \\
\cline { 2 - 2 } & Caminos Históricos e Itinerarios Culturales \\
\hline \multirow{3}{*}{ Específicas Imaginadas } & Literarias-cinematográficas-televisivas \\
\cline { 2 - 2 } & Personalidades históricas y personajes ficticios célebres \\
\cline { 2 - 2 } & Mágicas, esotéricas, míticas, legendarias \\
\hline Genéricas o mixtas & Mosaico de recursos (rutas y circuitos) \\
\hline
\end{tabular}

Tabla 3: Tipos y Subtipos de Rutas

Fuente: Hernández-Ramírez, Javier. Los caminos del patrimonio. Rutas turísticas e itinerarios culturales (2011)

La ruta que se presenta en este trabajo está basada en leyendas, milagros y mitos y según la tabla anterior se ajustaría al tipo "Especificas Imaginarias", concretamente al subtipo, "Mágicas, esotéricas, míticas y legendarias" Este tipo de rutas la mayoría de las veces presentan unas historias muy seductoras y atractivas, pero con poco fundamento histórico. Esto permite que el turista se trasporte a un lugar encantado, increíble y aunque muchas veces onírico, le permite descubrir el patrimonio desde otra perspectiva que permite normalmente una fácil comprensión del lugar visitado. Los espacios que recorren estas rutas son mostrados como insólitos y cargados de leyendas que refieren a acontecimientos, personajes históricos e incluso fantásticos combinados a veces con interpretaciones esotéricas (Hernández-Ramírez, 2011, Inglis y Holmes, 2003; Otamendi, 2008). Con este tipo de ruta, el turista puede transportarse a un lugar bucólico, donde vivir experiencias únicas. Según HernándezRamírez, Javier. 2011, es necesario para la realización de ésta tener en cuenta no solo los valores históricos sino también los valores identitarios y etnológicos como un conjunto articulado en el que participe activamente la sociedad local, analizando las tradiciones, las diferentes versiones de aquellas leyendas que han perdurado durante siglos, la forma de vida y el valor e importancia que les dan a éstas. La huella del pasado, su continuidad, es el argumento para la construcción de un territorio fosilizado, estancado, pero muy atractivo para la imaginación turística.

Como trasfondo central de la hipótesis de este trabajo debemos apostar por una concepción holística que integre los bienes arqueológicos, históricos, artísticos, ecológicos y etnológicos como un todo dentro de un contexto cultural y territorial determinado, basada en los usos y valores simbólicos autóctonos y esto es lo que intentamos hacer con esta propuesta. Es importante generar nuevas relaciones entre la población y su patrimonio a través de estas leyendas y misterios que forman parte de la idiosincrasia de éstos y ayudan a entender y comprender de una forma muy atractiva y complementaria el bien a la hora de interactuar con los visitantes. 


\subsection{Diseño de itinerario turístico: ruta turística "Morón Oculto"}

Como se ha señalado anteriormente, nuestra ruta trata de articular dos conjuntos de bienes patrimoniales, los que son resultado de tradiciones orales (leyendas) con los monumentos y bienes de interés etnológico artístico histórico.

A lo largo de la historia, Morón ha atesorado numerosos secretos, misterios, leyendas y milagros de una importante riqueza. Esta ruta va a posibilitar aunarlas todas para que el visitante viva una experiencia trascendental y lo valore como algo único e irrepetible, posibilitando que el turista interactúe a la vez que visita los diferentes monumentos de la localidad en los que acontecieron estas leyendas.

Todas estas leyendas van a contribuir a dar vida a una serie de monumentos de la localidad. Este bien diferenciador va a favorecer la posibilidad de ofrecer un producto único, con un interés mayor. El atractivo del bien crecerá con estas aportaciones que estudiosos y vecinos han contribuido para enriquecer aún más si cabe estas leyendas que, aunque no se saben si ocurrieron realmente, han influenciado notoriamente en el devenir histórico de esta ciudad y en la forma de ser de sus vecinos.

Estas leyendas invitan a adentrarse en un mundo de fantasía que te transportará a otras épocas y te ayudará a comprender mejor por qué aconteció de esa forma y cómo ha influenciado en el entorno. No podemos reducir una visita guiada a la descripción histórica artística de un bien, hay que ahondar más y ofrecer al visitante aquello que no puede no ver ni tocar, pero que también forma parte del bien.

La ruta turística "Morón Oculto" será un proyecto novedoso que servirá para dar a conocer aquellas leyendas, milagros y misterios que han llegado a nuestros días y que se desarrollaron en el entorno del pueblo, algunas ya relegadas a los libros y otras que ni siquiera están plasmadas en papel pero que han pasado de generación en generación. Como leyenda central se aprovecharía la fama y popularidad de la leyenda del Gallo conocida internacionalmente. Otras leyendas que tendrían cabida en este proyecto serían las siguientes: las leyendas del "caballo del escudo", la de la "conquista cristiana de la Fortaleza" o incluso otras que se remontan a la época romana como son la de "exportación de aceite de Oliva al mismo Cesar" y la de "las minas de Plata".

Para este proyecto de ruta hemos diseñado una imagen de marca y un logotipo con el que se identifique y sea reconocida a simple vista por el turista. A continuación, podemos ver el diseño realizado para la ruta "Morón Oculto":

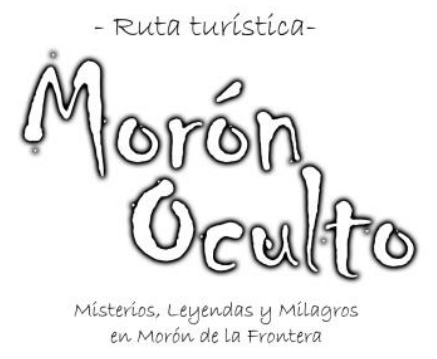

Figura 1: Logo y marca de la ruta "Morón Oculto" Fuente: Elaboración propia

Esta ruta está dirigida a todas aquellas personas interesadas en descubrir el rico patrimonio cultural de esta zona de la provincia de Sevilla, que posee un formidable legado artístico, monumental simbólico e inmaterial.

La presente ruta no pretende ser un manual de historia, sino que ha sido concebida básicamente con dos objetivos: por un lado remarcar las principales características artísticas y monumentales de este municipios, por el otro, abarcar en su totalidad todos aquellos elementos que constituyen el conjunto del patrimonio cultural de un pueblo, es decir, su arquitectura vernácula, sus celebraciones, su artesanía, su gastronomía y especialmente sus leyendas, misterios y milagros que componen en su conjunto el patrimonio etnológico.

La Ruta "Morón Oculto" va a permitir conocer, interpretar y experimentar desde una perspectiva holística el patrimonio cultural de la ciudad.

Para realizar de manera razonable el desarrollo de esta ruta hemos tenido en cuenta los siguientes procedimientos:

1. En primer lugar elaboramos un diagnóstico de los recursos culturales y turísticos de la localidad, analizando su demanda turística cultural, los recursos patrimoniales, el entramado institucional y si este mercado turístico es potencial. El objetivo del diagnóstico consiste en ofrecer una visión crítica de la situación, así como de sus posibilidades. 
2. En segundo lugar, consensuamos los objetivos y líneas estratégicas a desarrollar: nos hemos decantado por el diseño de una ruta cultural teniendo en cuenta que las rutas son una de las herramientas más activas y frecuentes utilizadas a la hora de organizar productos con base en el patrimonio. Permiten integrar en un producto elementos que individualmente no alcanzan un interés suficiente o que dificultan las tareas de promoción y comercialización. Esperamos que esta ruta proporcione una experiencia única que aumente la satisfacción del visitante, desde la experiencia principalmente educativa a la de simple disfrute.

3. En tercer lugar, conceptualizamos el criterio clave de interpretación, que permite convertir la visita en un descubrimiento, en una enriquecedora experiencia vivida. Estas claves de interpretación facilitan la difusión del destino, en general, y del patrimonio en particular. Hemos buscado que nuestra ruta interese al mayor número de público posible, intentando conseguir despertar sus curiosidades y también la necesidad de conocer y comprender. Este proyecto precisa de un plan de comercialización del destino y la configuración de los productos que vamos a ofertar.

4. Finalmente creímos necesaria la creación de una asociación cultural participada (moroneando) para el desarrollo turístico para facilitar una visión integrada, así como el seguimiento y evaluación de los efectos conseguidos por la puesta en marcha de esta ruta.

Este itinerario va dirigido a todo el público en general, pero puede suscitar especial interés a los amantes de la cultura, la historia y el patrimonio, teniendo en cuenta que nuestro público va a ser mayoritariamente nacional y en menor medida europeo.

Hemos creído conveniente para conseguir la difusión de esta ruta hacer uso de las nuevas tecnologías, además hemos considerado conveniente la realización de un díptico y de carteles divulgativos para la promoción exterior de la ruta.

Por último, hemos diseñado señalizaciones para las distintas leyendas que componen la ruta para facilitar la realización de ésta con una breve narración de éstas y de la repercusión que ha tenido a lo largo de la historia del municipio. Estas señales podrían colocarse llegando a un acuerdo con la administración local para armonizar la ruta en los principales puntos de ella.

Para complementar la ruta hemos creído necesario la realización de actividades complementarias para mostrar un mayor aliciente a los visitantes; entre ellas destacan la posibilidad de realizar representaciones teatrales y conciertos vinculados a la temática de la ruta.

Otra propuesta es la divulgación de la ruta en los colegios y la organización de actividades de animación cultural en las que participen los alumnos recreando algunas de las leyendas de la ruta. Para los más pequeños sería interesante la edición de un comic que escenifique estas leyendas y con el que trabajar en clase antes de hacer la ruta.

Para la creación de esta ruta hemos recopilado todas aquellas leyendas y milagros más conocidos e interesantes para dar a conocerlas y que aquellas más desconocidas no se pierdan y sigan pasando de padres a hijos. Una vez hecho esto, será necesaria la creación de una marca identificativa y un folleto.

A través de esta encrucijada de leyendas, misterios y milagros, pretendemos contar el devenir, la historia e idiosincrasia de este municipio y dar a conocer parte de su pasado de una forma entretenida y didáctica, para que generaciones venideras puedan conocer estas leyendas.

\subsection{Leyendas y misterios que conforman la ruta e itinerarios propuestos para su desarrollo}

El origen de algunas de las leyendas, a fuerza de repetirse han adquirido un protagonismo relevante, sus orígenes suelen ser a veces dudoso s y oscuros, con lo que se originan diversas interpretaciones y conjeturas, la mayoría deformadas de la realidad. Según García López, Juan José (2005), Las leyendas populares suelen tener siempre un vago fundamento histórico que las prestigia a través del tiempo y de generaciones sucesivas, aunque su verdadero origen escape muchas veces a la curiosidad de los eruditos.

A continuación, vamos a enumerar algunas de estas leyendas, misterios y milagros más relevantes estructurándolas en tres itinerarios tematizados aquellas leyendas que tienen más peso y están relacionadas entre sí y un cuarto itinerario que englobe los diferentes misterios y leyendas de Morón a lo largo de la Historia, desde las Hispania Romana hasta nuestros días, dando lugar a la ruta propuesta "Morón Oculto": 
Primer itinerario: Leyendas del Escudo y de la Toma de Morón.

Segundo itinerario: La Leyenda del Gallo de Morón.

Tercer itinerario: Misterios y Milagros en la Iglesia de San Miguel.

Cuarto itinerario: Ruta "Morón Oculto".

\begin{tabular}{|c|c|c|c|c|c|}
\hline \multirow{2}{*}{ Leyendas, misterios y milagros } & \multicolumn{4}{|c|}{ Itinerarios } & \multirow{2}{*}{ Cronología } \\
\hline & 10 & $2^{\circ}$ & 3 & $4^{\circ}$ & \\
\hline Minas de plata & & & & & Hispania Romana \\
\hline Aceite del emperador. & & & & & Hispania Romana \\
\hline El reino taifa de Morón & & & & & S.XI \\
\hline Santa Catalina y el escudo de Morón & & & & & S. XII \\
\hline Reconquista de Morón & & & & & S.XIII \\
\hline Pasadizos del Castillo lo ha escuchadd & & & & & S.XIII \\
\hline Torre de Algamazamara & & & & & S.XIII \\
\hline El Gallo de Morón & & & & & S.XVI \\
\hline Ajusticiamientos de la Inquisición & & & & & S.XVI \\
\hline El gigante de los Jesuita & & & & & S.XVII \\
\hline Milagro de San José & & & & & S.XVII \\
\hline Función del Rayo & & & & & S.XVIII \\
\hline El dedo de mármol & & & & & S.XIX \\
\hline Supersticiones de Fernando Villalón & & & & & S.XX \\
\hline La campanita de la muerte & & & & & Hasta nuestros días \\
\hline
\end{tabular}

Tabla 4: Tabla de Leyendas e itinerarios Fuente: Elaboración propia

Primer itinerario: Leyendas del Escudo y de la Toma de Morón: Este primer itinerario se centra en las zonas aledañas al castillo, ya que la mayoría de estas leyendas transcurren en este entorno. Por lo que este itinerario consistiría en un recorrido por las ruinas de la antigua fortificación árabe, de la que aún se conservan lienzos de murallas y la torre del homenaje.

Segundo itinerario: La Leyenda del Gallo de Morón: La leyenda del Gallo de Morón es la más famosa de todas las estudiadas. El Gallo de Morón es conocido internacionalmente y por ello le hemos dedicado uno de los itinerarios en exclusividad. El itinerario de esta ruta recorre los dos monumentos dedicados al gallo y los diferentes lugares donde transcurrieron los hechos, como el edificio del Cabildo (actual ayuntamiento) y la Peña del Moro.

Tercer itinerario: misterios y Milagros en la Iglesia de San Miguel Arcángel: En este tercer itinerario hemos englobado dos milagros y un misterio acontecidos en la Parroquia de San Miguel.

Cuarto itinerario: Ruta "Morón Oculto": Debido a la gran riqueza de leyendas, misterios y milagros en la ciudad de Morón de la Frontera, hemos aunado todas ellas en esta ruta titulada "Morón Oculto" y que ha sido el itinerario empleado para nuestro estudio en el que hemos desglosado todo el proceso para la realización y puesta en marcha de esta ruta.

\section{CONSIDERACIONES FINALES}

La cultura hoy día es una de las principales motivaciones que impulsan a los turistas a visitar un lugar determinado; no es un mero decorado. La cultura y el turismo deben ir de la mano, ambos convergen al mismo tiempo, por un lado la cultura se comercializa y por otro el turismo se culturaliza. Es necesaria una cooperación entre las áreas de Cultura y Turismo y creemos que los objetivos propuestos en este estudio lo han conseguido. 
En este estudio nos proponíamos alcanzar dos objetivos principales, y llegados a este punto consideramos que se ha conseguido este propósito, ya que a lo largo del trabajo hemos desarrollado detalladamente todo lo referente a la cultura como motor turístico y la riqueza patrimonial de Morón de la Frontera, esta ardua investigación y compilación de artículos para poder desarrollar ambos objetivos ha dado lugar a la realización de un inventario de la localidad y la creación de una nueva ruta turística.

En el inventario de los recursos patrimoniales de Morón, se ha tenido en cuenta el valor intrínseco del bien según sus usos y funciones, lo que ha permitido obtener una visión holística del patrimonio cultural de Morón.

Con respecto a la realización de una ruta asociada a misterios y leyendas de la localidad, esta va a permitir por un lado, añadir un mayor valor a los bienes patrimoniales, ya que incorpora los saberes e imaginarios tradicionales, estimulando un turismo lleno vivencias y experiencias y por otro lado poner en valor y recuperar la memoria de leyendas, misterios y milagros entre la propia población local.

Con esto conseguimos que el turista no solo visite edificios aislados, sino que a través de la ruta se integren diferentes espacios, superando la visión monumentalista que solo atiende a objetos aislados. Gracias a esta ruta se podrá visitar Morón como un conjunto, en el que las leyendas permitirán articular elementos aparentemente aislados dándoles contenido. El turista entrará en Morón a través de lo inmaterial, presentando la ciudad como algo singular.

En conclusión, el turismo puede contribuir al patrimonio, lo novedoso de este trabajo es la articulación entre un inventario y una ruta turística. Las leyendas constituyen el hilo conductor de las rutas, lo que da contenido integral al itinerario y se convierten en el pretexto para visitar monumentos y manifestaciones del patrimonio etnológico de la localidad.

La cultura es en definitiva un indiscutible motor de la actividad turística, que como hemos visto a lo largo de este trabajo ha permitido dar coherencia a una propuesta turística atractiva y auténtica que permite aproximarse al turista a la población local y sentir en primera persona aquello que está viendo desde una perspectiva holística.

\section{BIBLIOGRAFIA}

\section{Artículos en revistas:}

Agudo Torrico, Juan. (1999): "Patrimonio etnológico e inventarios: Inventarios para conocer, inventarios para intervenir", en "PATRIMONIO ETNOLÓGICO: NUEVAS PERSPECTIVAS DE ESTUDIO". Vol. № 1: pp. 52-69

Dacosta Martínez, Arsenio (2008): "Musealizar la tradición". Reflexiones sobre la representación pública del pasado", en "REVISTA DE ANTROPOLOGÍA EXPERIMENTAL". Vol. № 8: pp. 97-106.

Hernández Ramírez, Javier. (2011): Los caminos del patrimonio. Rutas turísticas e itinerarios culturales. En "PASOS. Revista de Turismo y Patrimonio Cultural". Vol. 9 № 2: pp. 225-236.

Limón Delgado, Antonio. (1999): "Patrimonio, ¿De quién?" en "PATRIMONIO ETNOLÓGICO: NUEVAS PERSPECTIVAS DE ESTUDIO". Vol. № 10: pp. 8-15.

Mckercher, B.; Ho, P.S.Y. (1999): "Managing Heritage Resources as Tourism Products" IN "ASIA PACIFIC JOURNAL OF TOURISM RESEARCH. Vol.9 № 3: pp. 255-266.

Moreno Navarro, Isidoro (2002): “Cultura andaluza, patrimonio cultural y políticas del patrimonio", en "Demófilo. Revista de Cultura Tradicional de Andalucía”. Vol. № 37: pp. 89-108.

Toselli Génova, Claudia. (2007): Algunas reflexiones sobre el turismo cultural. Universidad del Salvador (Argentina) en revista "PASOS. Revista de Turismo y Patrimonio Cultural". Vol. 4: pp. 175-182

\section{Trabajos presentados en congresos, jornadas:}

Ramos Campos, Germán. (2014) “Donde nace la cal”. Valor patrimonial y potencialidad Turística en Morón de la Frontera (Sevilla). Trabajo de Fin de Máster.

\section{Libros:}

Bohórquez Villalón, Antonio (1994): "Anales de Morón", [introducción, edición, notas e índices de Joaquín Pascual Barea]. Gráficas Olimpia. Morón de la Frontera.

Castellano de Torres, Ramón (1991): "Historia Ilustrada de Morón de la Frontera". Fundación Fernando Villalón. Morón de la Frontera. 
Castellano de Torres, Ramón (2001): “Monumentos de Morón”. Fundación Fernando Villalón. Morón de la Frontera.

Collantes de Terán Y Caamaño, Francisco (1990): "Historia de Morón de la Frontera". Fundación Fernando Villalón. Morón de la Frontera.

García López, Juan José (1982): "Crónicas para una Historia de Morón". Ayuntamiento de Morón. Morón de la Frontera.

Hernández Ramírez, Javier (2011): Tiempo de hornadas. Diputación Provincial. Huelva. Etnografía de la actividad calera en Santa la Real.

Morón de Castro, María Fernanda (1995): "La Iglesia de San Miguel: cinco siglos en la Historia de Morón de la Frontera (XIV-XVIII)". Fundación Fernando Villalón. Morón de la Frontera.

\title{
Capítulos de libros:
}

Camacho R, Raúl Alfonso (2006): "El turismo al servicio del patrimonio cultura". En "Memorias de VII Encuentro para la Promoción y Difusión del Patrimonio Inmaterial de los países Iberoamericanos" San Felipe. Venezuela: pags.280-284.

\section{Leyes, decretos y normativas:}

Ley 14/2007, del 26 de noviembre de Patrimonio Histórico de Andalucía.

Decreto 304/2009, del 14 Julio, en el que se inscribe en el Catálogo General de Patrimonio Histórico como Bien de Interés Cultural, con la categoría de Lugar de Interés Etnológico, el bien denominado Las Caleras de la Sierra, en Morón de la Frontera (Sevilla)

Normativa Unesco/2003, del 17 octubre París, para la salvaguardia del Patrimonio Cultural Inmaterial.

Ley 16/1985, de 25 de junio, del Patrimonio Histórico Español

\section{Recursos Webs:}

\author{
www.museocaldemoron.com \\ www.serraniasuroeste.org \\ www.consorcioviaverde.es \\ www2.unwto.org/es \\ www.iaph.es \\ www.personal.us.es/jhernan/ \\ www.unesco.org
}

